\title{
Association between random glucose and all-cause mortality: findings from the mortality follow-up of the German National Health Interview and Examination Survey 1998
}

\author{
Jens Baumert*, Christin Heidemann, Rebecca Paprott, Yong Du and Christa Scheidt-Nave
}

\begin{abstract}
Background: Random glucose is widely measured in epidemiological studies and in the clinical setting when standardized fasting protocols and oral glucose tolerance testing or $\mathrm{HbA}_{1 c}$ measuring are not feasible. The relationship between random glucose and all-cause mortality has hardly been studied so far and was examined in the present study.

Methods: We ascertained mortality status among 5955 persons aged 18-79years and free of known diabetes when participating in the German National Health Interview and Examination Survey 1998 (mean observation time 11.7 years, 458 deaths). Cox regression was applied to analyze the association of random serum glucose with allcause mortality taken potential confounders into account. Relative mortality risks were estimated as hazard ratios (HRs) with 95\% confidence intervals (Cls) modeling random glucose as categorical or continuous variable.

Results: Compared to random glucose levels of $4.3-<5.3 \mathrm{mmol} / \mathrm{L}$, HRs $(95 \% \mathrm{Cls})$ were $1.94(0.85-4.45)$ for levels $<4.3 \mathrm{mmol} / \mathrm{L}$ and 1.16 (0.89-1.50), $1.20(0.91-1.58), 1.42$ (0.88-2.29), 2.02 (1.26-3.25) and 4.71 (2.20-10.10) for levels $5.3-<5.8,5.8-<6.8,6.8-<7.8,7.8-<11.1$ and $\geq 11.1 \mathrm{mmol} / \mathrm{L}$, adjusted for age, sex, lifestyle, anthropometry and chronic diseases. An additional adjustment for fasting time or $\mathrm{HbA}_{1 c}$ yielded similar estimates. Modeling continuous random glucose by restricted cubic spline functions revealed comparable findings.

Conclusions: In the present epidemiological study drawn from the general population, random glucose showed a significant association with all-cause mortality, independent of main potential confounders. Thus, random glucose measures are highly relevant to health risk assessment among people without known diabetes when fasting glucose or $\mathrm{HbA}_{1 \mathrm{c}}$ are difficult to obtain.
\end{abstract}

Keywords: Random glucose, All-cause mortality, Fasting time, Diabetes prevention, Population-based study

\footnotetext{
* Correspondence: baumertj@rki.de

Department of Epidemiology and Health Monitoring, Robert Koch Institute,

General-Pape-Strasse 62-66, D-12101 Berlin, Germany
}

(c) The Author(s). 2018 Open Access This article is distributed under the terms of the Creative Commons Attribution 4.0 International License (http://creativecommons.org/licenses/by/4.0/), which permits unrestricted use, distribution, and reproduction in any medium, provided you give appropriate credit to the original author(s) and the source, provide a link to the Creative Commons license, and indicate if changes were made. The Creative Commons Public Domain Dedication waiver (http://creativecommons.org/publicdomain/zero/1.0/) applies to the data made available in this article, unless otherwise stated. 


\section{Background}

Diabetes and preceding states of hyperglycemia are major risk factors for cardiovascular disease (CVD) and mortality and highly prevalent among adults in Germany and worldwide [1-4]. However, findings on mortality risk vary according to the glycemic measure applied, as shown in a recent systematic review including a meta-analysis of 25 prospective studies among persons with prediabetes [5]. Numerous studies found strong increases in CVD mortality risk for elevated fasting glucose levels $[2,6,7]$. Studies investigating the association between $\mathrm{HbA}_{1 \mathrm{c}}$ and mortality risk among people without diagnosed diabetes have reported conflicting results, with most studies showing a J shaped association with increased risk at very low $\mathrm{HbA}_{1 \mathrm{c}}$ measures as well as at measures at $6.4 \%$ or above $[8,9]$.

Fasting glucose and $\mathrm{HbA}_{1 \mathrm{c}}$ are routine measures for glycemic status used for diagnosing diabetes; however, there is evidence that applying currently recommended diagnostic criteria for the diagnosis of previously unknown diabetes or prediabetes using fasting glucose and $\mathrm{HbA}_{1 \mathrm{c}}$ are not identifying the same people [10]. Furthermore, fasting glucose is rather difficult to obtain in large epidemiological studies and sometimes even in daily routine care, because individuals are often not in a fasting state at blood withdrawal. Moreover, different definitions for the fasting state are in use, especially in terms of fasting time, which may contribute to inconsistent glucose measurements and findings across studies [11]. $\mathrm{HbA}_{1 \mathrm{c}}$ assessment is connected with higher costs compared to glucose measurement.

Against this background, we examined whether random glucose as a less well-standardized but highly feasible measure is predictive of mortality from all causes among people without previously diagnosed diabetes. While it is well-known that random glucose is a strong predictor of incident diabetes [12, 13], the relationship to mortality has hardly been studied so far and did not include all-cause mortality as an outcome [14, 15]. Therefore, we asked: (1) Is there a significant relationship independent of potential confounders? and (2) Do fasting time and $\mathrm{HbA}_{1 \mathrm{c}}$ contribute to explain the relationship between random glucose and mortality?

\section{Methods}

\section{Study design and setting}

The present analysis is based on the mortality follow-up of the German National Health Interview and Examination Survey 1998 (GNHIES98) sample. The GNHIES98 was conducted by the Robert Koch Institute between October 1997 and March 1999 and included 7124 adults representative of the 18 to 79 -year-old residential, non-institutionalized population in Germany. Details of the study design, sampling procedure, response rates and data collection have been published previously [16].
In brief, a two-stage cluster sampling procedure was applied for the selection of survey participants (response rate: $61.4 \%$ ). In the first stage of sampling, sample points (i.e. study locations) reflecting community sizes and structures in Germany were drawn and in the second stage, random samples stratified by sex and age were selected from local population registries proportional to the sex and age structure of the population in Germany.

The survey included a standardized computer-assisted personal interview (CAPI) administered by specifically trained study physicians, a standardized self-administered questionnaire which was checked by trained interviewers for plausibility and completeness, and a physical examination, including standardized anthropometric measurements and blood sampling as well as a detailed medication review conducted by trained health professionals. Blood samples were drawn over the day and processed within one hour and stored at $-40{ }^{\circ} \mathrm{C}$ until analysis in the central laboratory unit at the Robert Koch Institute. Any medications taken in the past 7 days were recorded using the unique medication identifiers ("Pharmazentralnummer", PZN) on the original medication containers brought to the study center by study participants for this purpose. The PZN was used for medication coding according to the WHO "Anatomical-Therapeutic-Chemical" classification system (ATC-Code).

The vital status could be assessed for 6979 among the 7124 GNHIES98 participants $(98.0 \%)$ by the mortality follow-up as previously described in detail [17]. Briefly, all GNHIES98 participants who had agreed to follow-up contacts were recontacted between October 2008 and October 2011 and invited to participate in the first wave of the German Health Interview and Examination Survey for Adults (DEGS1). For participants who did not respond to the invitation, vital status was obtained from local population registries including the date of death for deceased individuals. Surviving GNHIES98 participants were censored at the last date of contact, i. e. the date of contact to the population registry for non-respondents, the date of refusal for those who actively declined participation in DEGS1, or the date of DEGS1 participation for those who also took part in DEGS1.

The GNHIES98 and its mortality follow-up were approved by the Federal Office for the Protection of Data (Germany). All participants provided written informed consent before enrolment.

\section{Study population}

Among 7124 participants of the GNHIES98 study, a number of 6750 individuals reporting no history of physician-diagnosed diabetes and no use of antidiabetic medication within the last seven days preceding the interview were defined as free of known diabetes at the baseline examination and comprised the source 
population of the present study. After sequential exclusion of participants with no information regarding vital status $(n=138)$, known diabetes $(n=25)$, random glucose level or fasting time $(n=336)$ or main covariates used in the present analyses $(n=299)$, the final study population consisted of 5955 participants (2919 men and 3036 women) aged $18-79$ years at baseline.

\section{Assessment of random glucose and fasting time}

Random glucose was determined by standardized measures of serum glucose drawn from fresh whole blood specimens randomly taken over the day using glukose-oxidase-peroxidase-4-aminophenazon-phenol by a MEGA analyzer (Merck, Darmstadt, Germany). For analyses as categorical variable, random glucose level was classified in seven categories $(<4.3,4.3-<5.3,5.3-<5.8$, $5.8-<6.8,6.8-<7.8,7.8-<11.1$ and $\geq 11.1 \mathrm{mmol} / \mathrm{L})$ as previously suggested [14]. The two highest categories followed classifications concerning oral glucose tolerance 2-h postload thresholds for assessing diabetes and impaired glucose tolerance [4].

Fasting time was defined as the difference between time of drawing blood specimen and time since the last meal was taken, the latter based on self-report. For specific analyses, fasting time was classified into the categories $<2,2-<4,4-<8,8-<12$ and $\geq 12 \mathrm{~h}$.

\section{Assessment of covariates}

Covariates were chosen a priori to control for potential confounding of the relationship between random glucose and all-cause mortality. Age, sex, educational level, smoking status, alcohol intake and physical activity were obtained by self-administered questionnaire, anthropometric measures by physical examination, history of chronic diseases by CAPI and $\mathrm{HbA}_{1 \mathrm{c}}$ by blood sampling.

Educational level was assessed by the Comparative Analysis of Social Mobility in Industrial Nations (CASMIN) instrument, encompassing general as well as vocational training, and classified into the categories low, medium or high [18].

Smoking status was categorized as never, former and current smoking. Alcohol intake (g/day) was obtained by a semi-quantitative food frequency questionnaire [19] and classified into no, moderate $(>0-<20 \mathrm{~g} /$ day in men, $>0-<10 \mathrm{~g} /$ day in women) and high $(\geq 20 \mathrm{~g} /$ day in men, $\geq 10 \mathrm{~g} /$ day in women) alcohol intake. Physical activity was assessed by five categories as no sport, $<1 \mathrm{~h} /$ week, regularly $1-2 \mathrm{~h} /$ week, regularly $2-4 \mathrm{~h} /$ week, or regularly $>4 \mathrm{~h} /$ week. This information was aggregated into two categories $(<2 \mathrm{~h} /$ week or $\geq 2 \mathrm{~h} /$ week $)$ for the present analysis. Body mass index (BMI) was calculated as the ratio of body weight $(\mathrm{kg})$ and height squared $\left(\mathrm{m}^{2}\right)$. History of myocardial infarction, stroke, cancer, hypertension and hyperlipidemia were chosen as chronic diseases and were each defined as no or yes.

$\mathrm{HbA}_{1 \mathrm{c}}$ was measured in fresh whole blood specimens with a Diamat high-performance liquid chromatography analyzer (Bio-Rad Laboratories, Munich, Germany) and reagents of Recipe (Recipe Chemicals and Instruments, Munich, Germany) in the Robert Koch Institute Central Epidemiological Laboratory [20]. For specific analyses, $\mathrm{HbA}_{1 \mathrm{c}}$ level was classified into $<5.0,5.0-<5.7,5.7$ $-<6.5$ and $\geq 6.5 \%$ as previous studies showed a $U$ form relation of $\mathrm{HbA}_{1 \mathrm{c}}$ and all-cause mortality $[8,21]$.

\section{Statistical analyses}

Unadjusted analyses were performed by the Rao-Scott $x^{2}$ test for associations between random glucose categories and categorical variables and by the $F$ test for mean differences for continuous and (approximately) normally distributed variables across random glucose categories. Spearman correlation was applied to assess a potential monotonic relationship between continuous random glucose and fasting time. To display distribution measures of continuous random glucose across five fasting time categories, a Box plot was created. Crude mortality rates were calculated by dividing the number of deaths by the number of person-years observed within each random glucose category.

Adjusted analyses were performed by Cox proportional hazards regression models to estimate random glucose-specific hazard ratios (HRs) including 95\% confidence intervals $(95 \% \mathrm{CIs})$ for all-cause mortality during follow-up. As basic model, model 1 was adjusted for age (continuous) and sex. Further adjustments were made for the sociodemographic factor educational level in model 2, additionally for the lifestyle factors smoking, alcohol consumption and physical activity as well as the anthropometric factors BMI (continuous) and waist circumference (continuous) in model 3, and additionally for the five chronic diseases history of myocardial infarction, stroke, cancer, hypertension and hyperlipidemia in model 4 .

First, random glucose was included in the Cox regression models as categorical variable using a level of 4.3 $-<5.3 \mathrm{mmol} / \mathrm{L}$ as reference. Secondly, random glucose was included as continuous variable in the Cox regression and modelled by a spline regression approach applying restricted cubic spline functions with four knots set at the 5th, 25th, 75th and 95th percentile and choosing the median random glucose level of $5.2 \mathrm{mmol} / \mathrm{L}$ as reference.

Sensitivity analyses were performed to assess the stability of the main analyses and comprised a) an additional adjustment for fasting time $(<2,2-<4,4-<8$, $8-<12, \geq 12 \mathrm{~h}$ ), b) an exclusion of participants with fasting time $<2 \mathrm{~h}, \mathrm{c})$ an additional adjustment for $\mathrm{HbA}_{1 \mathrm{c}}$ $(<5.0,5.0-<5.7,5.7-<6.5$ and $\geq 6.5 \%)$, d) an exclusion of participants with $\mathrm{HbA}_{1 \mathrm{c}} \geq 6.5 \%$, e) an exclusion of 
participants with follow-up time $\leq 2$ years and, finally, f) an analysis in the source population $(n=6750)$ with multiple imputation of missing values using the "Fully Conditional Specification" method [22]. Furthermore, interaction analyses were carried out to examine potential modifications of the random glucose-mortality association by age, BMI and waist circumference (all continuous) and sex with adding the respective interaction terms (random glucose $\mathrm{x}$ modifier) to the Cox regression model 4 (described above).

Statistical analyses were performed by the statistical software package SAS version 9.4 (SAS Institute, Cary, NC, USA) using the survey procedures SURVEYMEANS, SURVEYFREQ and SURVEYPHREG to account for the complex survey design, except for analyses regarding Spearman correlation and spline modelling in the Cox regression (see below). The analyses included a survey weight accounting for deviations of the study population from the population in Germany as of December 31, 1997 within strata of sex, age, education, nationality, community size, federal state, and east/west Germany which may compensate under- or over-represented groups within these strata. Spearman correlation analyses were unweighted using CORR and spline modelling in the Cox regression was performed by PHREG which permitted consideration of weights but not accounting for cluster sampling. $P$ values $<0.05$ were considered to indicate statistical significance. The study followed the STROBE guidelines for cohort studies [23].

\section{Results}

\section{Description of study population}

Random glucose had a median level of $5.2 \mathrm{mmol} / \mathrm{L}$ and ranged from 2.6 to $20.6 \mathrm{mmol} / \mathrm{L}$. Table 1 shows the distribution of baseline characteristics across seven random glucose categories. Participants with higher random glucose categories were significantly more often older, male, low educated, obese, current smokers, high alcohol consumers, physically inactive and reported more often a history of myocardial infarction, stroke, hypertension and hyperlipidemia compared to participants with lower random glucose categories.

\section{Association of random glucose and fasting time}

Median fasting time was $5.4 \mathrm{~h}$ with lower quartile $3.8 \mathrm{~h}$ and upper quartile $8.6 \mathrm{~h}$. Continuous random glucose level and fasting time were weakly and non-monotonically correlated (Spearman's $\rho=-0.044$ ) as shown by a scatter plot (Fig. 1).

A Box plot revealed comparable distribution measures for random glucose levels across five fasting time categories (Additional file 1: Figure S1); slightly higher $=$ median random glucose $(5.4 \mathrm{mmol} / \mathrm{L})$ and higher variation in glucose measures was observed among persons with lowest fasting time $(<2 \mathrm{~h})$ as compared to those with fasting times between $2-<4 \mathrm{~h}$ and $\geq 12 \mathrm{~h}$.

\section{Association of random glucose and all-cause mortality}

A total number of 458 deaths (men: 287, women: 171) was observed over a mean follow-up time of 11.7 years (standard error 0.1). Crude mortality rates per 1000 person-years ranged from 3.8 in the lowest to 41.2 in the highest random glucose category (Table 2).

Compared to participants with random glucose levels in the reference category $(4.3-<5.3 \mathrm{mmol} / \mathrm{L})$, the ageand sex-adjusted relative mortality risk estimated by Cox regression was significantly increased for participants in the lowest random glucose category $(<4.3 \mathrm{mmol} / \mathrm{L})$ as well as for those with random glucose levels in the two highest categories (model 1, Table 1) which persisted after further adjustment for educational level, lifestyle and anthropometry (model 3). Additional adjustment for major chronic diseases attenuated the relative mortality risk (HR: 1.94, 95\% CI: 0.85-4.45) for participants in the lowest random glucose category to non-significance, but not for those in the higher categories with HRs (95\% CI) of $1.16(0.89-1.50), 1.20(0.91-1.58), 1.42$ (0.88-2.29), $2.02(1.26-3.25)$ and $4.71(2.20-10.10)$ for levels of $5.3-<5.8$ to $\geq 11.1 \mathrm{mmol} / \mathrm{L}$ (model 4 ).

Modeling continuous random glucose levels by restricted cubic spline functions in the Cox regression models revealed a similar shape of the random glucose-mortality association as shown for model 4 (Fig. 2). However, the confidence intervals at the tails of the hazard ratios were wide due to the low number of participants with extreme random glucose values.

\section{Sensitivity analyses}

Additional adjustment for fasting time in model 4 or excluding participants with fasting time $<2 \mathrm{~h}$ did not change estimates substantially (Table $3 \mathrm{a}$ and $\mathrm{b}$ ). Hazard ratios for the two highest categories were attenuated but remained significantly increased when additionally controlling for $\mathrm{HbA}_{1 \mathrm{c}}$ (Table 3c). After excluding participants with $\mathrm{HbA}_{1 \mathrm{c}} \geq 6.5 \%$, no more observations in the highest random glucose category were observed; however, the relative risk of death was significantly increased among persons with random glucose levels of $7.8-<11.1 \mathrm{mmol} / \mathrm{L}$ (Table 3d). Comparable estimates as in the main analyses were obtained when participants with follow-up time $\leq 2$ years were excluded (Table $3 e$ ). Performing the main analyses in the source population $(n=6750)$ with imputation of missing variable information estimated significant hazard ratios for the three highest categories; the hazard ratio for random glucose level $\geq 11.1 \mathrm{mmol} / \mathrm{L}$ was lower than in the analyses using the study population (2.96 versus 4.71 ) (Table $3 f)$.

\section{Interaction analyses}

No significant modifications in the association of random glucose level category and all-cause mortality was 
Table 1 Baseline characteristics of the study population by random glucose categories

\begin{tabular}{|c|c|c|c|c|c|c|c|c|}
\hline \multirow[b]{2}{*}{ Characteristic } & \multicolumn{8}{|c|}{ Random glucose category (in $\mathrm{mmol} / \mathrm{L}$ ) } \\
\hline & $<4.3$ & $4.3-<5.3$ & $5.3-<5.8$ & $5.8-<6.8$ & $6.8-<7.8$ & $7.8-<11.1$ & $\geq 11.1$ & $p$ value \\
\hline N & 224 & 3,105 & 1,510 & 843 & 161 & 93 & 19 & - \\
\hline Age class (\%) & & & & & & & & $<0.001$ \\
\hline $18-39$ years & 74.6 & 52.2 & 34.9 & 23.7 & 26.3 & 14.4 & 11.8 & \\
\hline 40-49 years & 15.2 & 19.9 & 18.5 & 18.7 & 10.6 & 14.0 & 21.8 & \\
\hline $50-59$ years & 6.5 & 14.4 & 20.1 & 19.9 & 27.9 & 23.3 & 25.1 & \\
\hline $60-69$ years & 2.2 & 9.1 & 15.4 & 24.1 & 21.1 & 28.3 & 23.8 & \\
\hline $70-79$ years & 1.5 & 4.5 & 11.0 & 13.6 & 14.1 & 20.0 & 17.5 & \\
\hline Age (years) & $33.4(1.1)$ & $41.1(0.4)$ & $48.0(0.6)$ & $51.9(0.7)$ & $52.1(1.6)$ & $57.4(1.8)$ & $56.5(2.7)$ & $<0.001$ \\
\hline Male sex (\%) & 42.6 & 45.3 & 54.0 & 59.3 & 60.6 & 60.2 & 56.9 & $<0.001$ \\
\hline Educational level (\%) & & & & & & & & $<0.001$ \\
\hline Low & 42.8 & 43.3 & 50.8 & 58.2 & 55.6 & 62.8 & 72.7 & \\
\hline Medium & 42.8 & 44.3 & 36.6 & 30.1 & 35.0 & 30.9 & 16.3 & \\
\hline High & 14.5 & 12.4 & 12.6 & 11.7 & 9.4 & 6.3 & 11.0 & \\
\hline BMI category $(\%)^{2}$ & & & & & & & & $<0.001^{\mathrm{a}}$ \\
\hline$<25 \mathrm{~kg} / \mathrm{m}$ & 64.1 & 47.5 & 34.2 & 26.2 & 17.7 & 19.3 & n.a. ${ }^{a}$ & \\
\hline $25-<30 \mathrm{~kg} / \mathrm{m}^{2}$ & 28.9 & 36.2 & 43.5 & 45.7 & 50.4 & 29.1 & 35.7 & \\
\hline$\geq 30 \mathrm{~kg} / \mathrm{m}^{2}$ & 6.9 & 16.2 & 22.3 & 28.1 & 31.9 & 51.5 & 64.3 & \\
\hline BMI $\left(\mathrm{kg} / \mathrm{m}^{2}\right)$ & $24.1(0.3)$ & $25.9(0.1)$ & $27.1(0.1)$ & $27.9(0.2)$ & $28.7(0.4)$ & $30.5(0.7)$ & $32.6(1.4)$ & $<0.001$ \\
\hline Waist circumference category $(\%)^{b}$ & & & & & & & & $<0.001$ \\
\hline Normal & 65.6 & 50.5 & 35.5 & 27.9 & 22.3 & 17.0 & 2.5 & \\
\hline Moderate & 22.6 & 24.1 & 27.1 & 26.7 & 27.5 & 21.3 & 10.0 & \\
\hline High & 11.9 & 25.4 & 37.4 & 45.4 & 50.2 & 61.6 & 87.5 & \\
\hline \multicolumn{9}{|l|}{ Waist circumference $(\mathrm{cm})^{c}$} \\
\hline Men & $88.9(1.1)$ & $94.4(0.4)$ & $96.7(0.4)$ & $99.1(0.6)$ & $100.1(1.8)$ & $102.0(1.5)$ & $109.8(3.2)$ & $<0.001$ \\
\hline Women & $78.4(1.1)$ & $81.8(0.4)$ & $86.8(0.6)$ & $90.0(0.8)$ & $91.5(1.8)$ & $99.4(2.4)$ & $105.9(3.4)$ & $<0.001$ \\
\hline Smoking (\%) & & & & & & & & $<0.001$ \\
\hline Never smoker & 48.3 & 36.9 & 30.4 & 28.7 & 27.4 & 24.9 & 30.3 & \\
\hline Former smoker & 12.9 & 19.4 & 23.4 & 25.7 & 27.5 & 23.9 & 20.0 & \\
\hline Current smoker & 38.8 & 43.7 & 46.2 & 45.7 & 45.1 & 51.2 & 49.7 & \\
\hline Alcohol consumption (\%) ${ }^{d}$ & & & & & & & & $<0.001$ \\
\hline No & 21.7 & 18.4 & 18.2 & 18.2 & 16.5 & 27.9 & 9.0 & \\
\hline Moderate & 69.8 & 63.9 & 59.2 & 59.7 & 57.7 & 49.9 & 52.4 & \\
\hline High & 8.5 & 17.7 & 22.6 & 22.1 & 25.7 & 22.2 & 38.6 & \\
\hline Physical activity < 2 h/week (\%) & 72.8 & 78.9 & 80.9 & 83.3 & 84.6 & 88.2 & 96.5 & 0.003 \\
\hline \multicolumn{9}{|l|}{ History of chronic diseases } \\
\hline Myocardial infarction & 0.6 & 1.2 & 1.6 & 3.6 & 5.2 & 3.6 & 3.7 & $<0.001$ \\
\hline Stroke & 0.4 & 0.6 & 1.2 & 1.0 & 0.6 & 6.4 & 4.0 & $<0.001$ \\
\hline Cancer & 2.5 & 2.8 & 3.2 & 3.6 & 3.5 & 5.7 & 4.0 & 0.682 \\
\hline Hypertension & 4.9 & 14.6 & 24.2 & 32.6 & 35.6 & 54.0 & 48.7 & $<0.001$ \\
\hline Hyperlipidemia & 8.5 & 17.9 & 25.7 & 28.8 & 33.2 & 38.6 & 55.5 & $<0.001$ \\
\hline
\end{tabular}

Information is given as arithmetic mean (standard error) or percentage. Differences in means or percentages by random glucose categories were assessed by $F$ test or Rao-Scott $X^{2}$ test

${ }^{a}$ no observations in this cell; $\mathrm{p}$ value for association excluding random glucose level $\geq 11.1 \mathrm{mmol} / \mathrm{L}$

${ }^{b}$ normal / moderate / high: $<94 / 94-<102 / \geq 102 \mathrm{~cm}$ in men and $<80 / 80-<88 / \geq 88 \mathrm{~cm}$ in women

c mean (standard error) is given separately for men and women due to sex-specific differences in waist circumference distribution

${ }^{d}$ no / moderate / high: $0 />0-<20 / \geq 20 \mathrm{~g} /$ day in men and $0 />0-<10 / \geq 10 \mathrm{~g} /$ day in women 


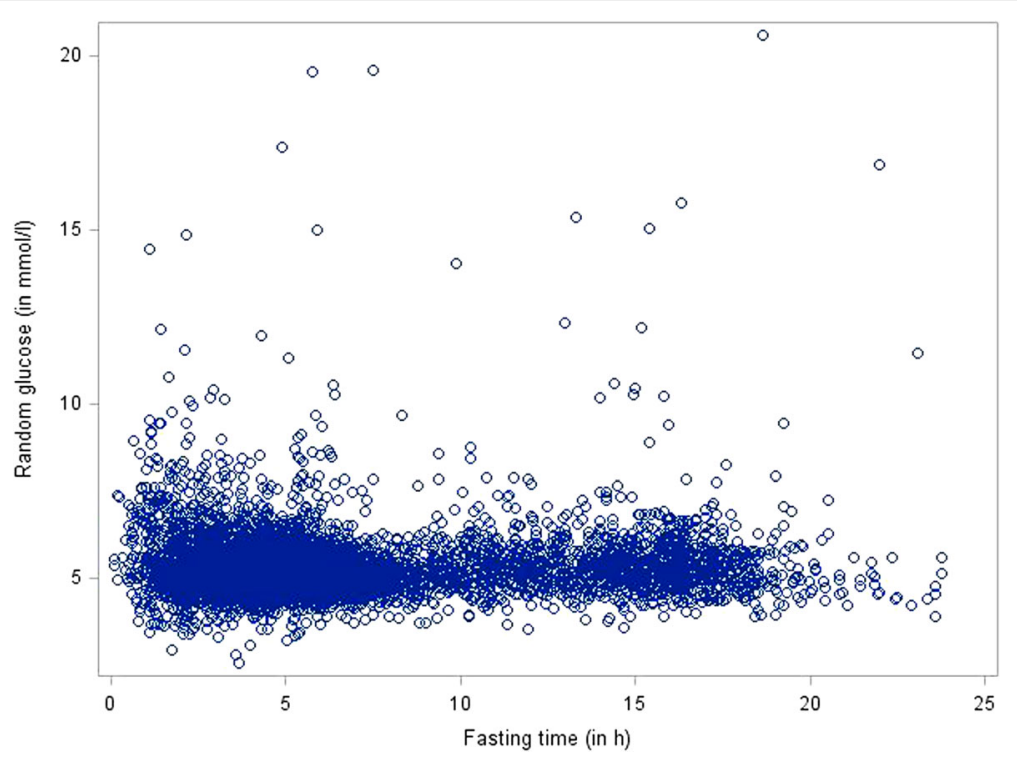

Fig. 1 Correlation of random glucose and fasting time, displayed as scatter plot

observed by age, sex and BMI in interaction analyses ( $\mathrm{p}$ for interactions $0.208,0.744$ and 0.108 ). However, a significant interaction was estimated regarding waist circumference ( $p$ for interaction 0.011 ); the association was more pronounced in participants with higher than with lower waist circumference levels. Modelling random glucose level by restricted cubic spline functions revealed no significant interaction by age, sex, BMI or waist circumference ( $p$ for interactions $0.133,0.156,0.802$ and 0.410 ).

\section{Discussion}

\section{Overall findings}

In the present epidemiological study drawn from the general population, random glucose was significantly associated with all-cause mortality, as an increase in mortality risk was found in participants with very low and high random glucose levels which was independent from main mortality-related risk factors as well as from fasting time and $\mathrm{HbA}_{1 \mathrm{c}}$. Changes in the effects of random glucose levels on mortality risk were rather low when adjusting for these factors. As far as we know, this is the first investigation about the association of random glucose and all-cause mortality in a study population drawn from the general population.

\section{Association of random glucose and all-cause mortality}

The present study investigated the association of random glucose with all-cause mortality, in contrast to a variety of previous studies which examined other endpoints. A large study based on a population-based sample of Chinese adults found a monotonically increasing CVD mortality risk using the same random glucose

Table 2 Mortality rate and risk for all-cause mortality (HR $(95 \% \mathrm{CI}))$ by random glucose categories

\begin{tabular}{|c|c|c|c|c|c|c|c|}
\hline & \multicolumn{7}{|c|}{ Random glucose category (in mmol/L) } \\
\hline & $<4.3$ & $4.3-<5.3$ & $5.3-<5.8$ & $5.8-<6.8$ & $6.8-<7.8$ & $7.8-<11.1$ & $\geq 11.1$ \\
\hline N & 224 & 3105 & 1510 & 843 & 161 & 93 & 19 \\
\hline $\mathrm{N}$ of deaths & 10 & 158 & 124 & 110 & 27 & 22 & 7 \\
\hline Crude $\mathrm{MR}^{\mathrm{a}}$ & 3.8 & 4.0 & 8.1 & 11.8 & 13.4 & 25.8 & 41.2 \\
\hline Model 1 & $2.46(1.12-5.38)$ & ref. & $1.06(0.84-1.34)$ & $1.18(0.93-1.51)$ & $1.34(0.83-2.15)$ & $1.85(1.15-2.98)$ & $4.36(2.00-9.50)$ \\
\hline Model 2 & $2.43(1.11-5.34)$ & ref. & $1.06(0.84-1.35)$ & $1.18(0.93-1.51)$ & $1.29(0.80-2.08)$ & $1.77(1.08-2.90)$ & $4.45(2.07-9.55)$ \\
\hline Model 3 & $2.11(1.00-4.43)$ & ref. & $1.14(0.90-1.46)$ & $1.22(0.94-1.59)$ & $1.43(0.90-2.26)$ & $1.79(1.07-3.01)$ & $4.42(2.14-9.11)$ \\
\hline Model 4 & $1.94(0.85-4.45)$ & ref. & $1.16(0.89-1.50)$ & $1.20(0.91-1.58)$ & $1.42(0.88-2.29)$ & $2.02(1.26-3.25)$ & $4.71(2.20-10.10)$ \\
\hline
\end{tabular}

${ }^{a}$ MR: crude mortality rate per 1000 person-years

model 1: adjusted for age and sex

model 2: adjusted for age, sex and educational level

model 3: adjusted for age, sex, educational level, body mass index, waist circumference, smoking, alcohol consumption and physical activity

model 4: adjusted for age, sex, educational level, body mass index, waist circumference, smoking, alcohol consumption, physical

activity, myocardial infarction, stroke, cancer, hypertension and hyperlipidemia 


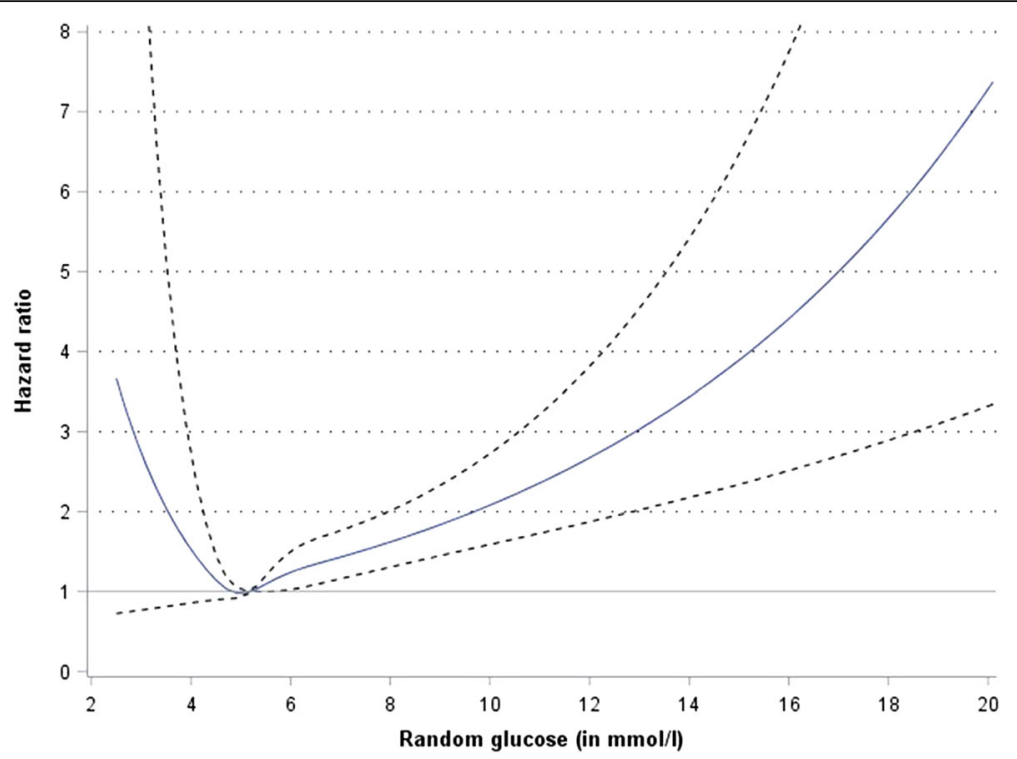

Fig. 2 Risk for all-cause mortality $(95 \%$ Cl) by continuous random glucose level, estimated by Cox regression and applying restricted cubic spline functions for modelling random glucose level

categories [14]; in contrast to our study with endpoint all-cause mortality, CVD mortality risks here were almost equal in the two lowest categories. Rosness et al. studied the association of random glucose (categorical and continuous) with dementia-related mortality in two study populations from Norway with different age ranges (35-49 and 65-80 years) and observed a relation in the younger but not in the older age group [15]; random glucose was categorized in quintiles as well as modelled with spline functions.

Both studies above did not show a U or J shaped association which may be due to different mortality endpoints (CVD or dementia-related in contrast to all-causes). However, in a large collaborative investigation including 48 studies with about 33,000 cases of death, a J shaped association of fasting glucose and death from all causes was reported in subjects without known diabetes and known pre-existing cardiovascular disease at baseline [2]. In a previous study regarding $\mathrm{HbA}_{1 \mathrm{c}}$ as another glycemic marker using the same data source (GNHIES98) as in the present analyses, Paprott et al. revealed a $\mathrm{U}$ shaped $\mathrm{HbA}_{1 \mathrm{c}}$-mortality relation when $\mathrm{HbA}_{1 \mathrm{c}}$ was modelled with spline functions in subjects without known diabetes indicating that very low $\mathrm{HbA}_{1 \mathrm{c}}$ levels are associated with increased risk for all-cause mortality, additionally to high $\mathrm{HbA}_{1 \mathrm{c}}$ levels ( $\geq 6.4 \%$ ) [8]. This is in line with findings of a meta-analysis of observational studies which indicated a J shaped relationship between $\mathrm{HbA}_{1 \mathrm{c}}$ levels and all-cause mortality in the non-diabetic as well as in the diabetic population [9].

Table 3 Sensitivity analyses for risk for all-cause mortality (HR $(95 \% \mathrm{Cl}))$ by random glucose categories

\begin{tabular}{llllllll}
\hline \multicolumn{7}{c}{ Model } & \multicolumn{7}{l}{ Random glucose category (in mmol/L) } \\
\cline { 2 - 8 } & $<4.3$ & $4.3-<5.3$ & $5.3-<5.8$ & $5.8-<6.8$ & $6.8-<7.8$ & $7.8-<11.1$ & $\geq 11.1$ \\
\hline a) adjusted for fasting time ${ }^{\mathrm{a}}$ & $1.96(0.87-4.43)$ & ref. & $1.16(0.89-1.51)$ & $1.22(0.92-1.60)$ & $1.46(0.89-2.39)$ & $2.10(1.28-3.42)$ & $4.99(2.33-10.73)$ \\
b) excluding fasting time $<2 \mathrm{~h}^{\mathrm{b}}$ & $2.07(0.85-5.03)$ & ref. & $1.14(0.87-1.50)$ & $1.18(0.89-1.57)$ & $1.43(0.85-2.41)$ & $2.20(1.25-3.86)$ & $4.38(2.01-9.58)$ \\
c) adjusted for $\mathrm{HbA}_{1 c}{ }^{c}$ & $1.96(0.86-4.44)$ & ref. & $1.17(0.90-1.51)$ & $1.21(0.91-1.60)$ & $1.35(0.83-2.20)$ & $1.82(1.09-3.03)$ & $3.46(1.49-8.04)$ \\
d) excluding $\mathrm{HbA}_{1 c} \geq 6.5 \%^{\mathrm{d}}$ & $1.73(0.73-4.09)$ & ref. & $1.12(0.85-1.48)$ & $1.23(0.92-1.63)$ & $1.08(0.54-2.13)$ & $2.46(1.50-4.03)$ & - \\
e) excluding FU time $\leq 2$ years $^{\mathrm{e}}$ & $2.10(0.87-5.06)$ & ref. & $1.12(0.85-1.46)$ & $1.11(0.82-1.48)$ & $1.30(0.77-2.19)$ & $1.97(1.21-3.21)$ & $3.72(1.51-9.19)$ \\
f) multiple imputation $^{f}$ & $1.68(0.92-3.07)$ & ref. & $1.12(0.88-1.41)$ & $1.25(0.98-1.59)$ & $1.81(1.17-2.80)$ & $1.94(1.26-3.01)$ & $2.96(1.48-5.92)$ \\
\hline
\end{tabular}

Models with adjustment as in model 4 with additional adjustment for fasting time in a) and $\mathrm{HbA}_{1 \mathrm{c}}$ in $\mathrm{c}$ )

a fasting time with five categories $(<2,2-<4,4-<8,8-<12$ and $\geq 12 \mathrm{~h})$

${ }^{\mathrm{b}} n=5579$ (428 cases, 5151 non-cases)

${ }^{c} \mathrm{HbA}_{1 \mathrm{c}}$ with five categories (<5.0, 5.0- $\left.<5.7,5.7-<6.5, \geq 6.5 \%\right)$

${ }^{\mathrm{d}} n=5718$ (386 cases, 5332 non-cases), no participant with random glucose $\geq 11.1 \mathrm{mmol} / \mathrm{L}$

e $n=5912$ (415 cases, 5497 non-cases)

$\mathrm{f} n=6570$ (551 cases, 6061 non-cases, 138 missings), missings were replaced in the source population using multiple imputation by fully conditional specification 
The association of random glucose and all-cause mortality was independent of main other mortality-related risk factors. The changes in the hazard ratios when sociodemographic and lifestyle factors as well as chronic diseases were taken into account was rather low in the two highest random glucose categories indicating no substantial confounding in the effects on all-cause mortality by these factors. In contrast, the effect change for the lowest random glucose category $(<4.3 \mathrm{mmol} / \mathrm{L})$ by these adjustments was more pronounced especially when chronic diseases were included in the regression model. Thus, the increased mortality risk in the very low compared to the reference random glucose group may partly be explained by these unfavourable conditions as previously found for low $\mathrm{HbA}_{1 \mathrm{C}}$ levels [8]. However, as the number of events in the lowest random glucose category was only 10 , this finding should not be overinterpreted; larger studies are necessary to examine the relation of very low random glucose levels and all-cause mortality in more detail.

Our interaction analyses suggest that an increased mortality risk in low and high random glucose levels was more pronounced in participants with higher compared to lower waist circumference levels. Previously, an increased risk for all-cause mortality has been shown for elevated levels of waist circumference in a population-based study of Canadian adults [24]. Considering this finding in the face with our interaction analysis may indicate that especially a combination of unfavourable levels in random glucose (low and high) and waist circumference (elevated) is related to an increased mortality risk which was surprising and may deserve further attention in future and larger studies.

However, the low case numbers at both tails of the random glucose level distribution should be kept in mind here and therefore interpretation regarding the interaction findings in the present study should be taken with caution.

\section{Random glucose and fasting time}

In the present study, the correlation between random glucose and fasting time was weak (correlation coefficient - 0.04); the distribution of random glucose was rather comparable across five fasting time categories, however a non-monotonic and suggestive J shaped association could be observed as shown in Additional file 1: Figure S1. This finding may be initially surprising as considerable more pronounced differences would be expected. However, Moebus et al. reported mean random glucose values of $5.3 \mathrm{mmol} / \mathrm{L}$ at zero hours, $5.2 \mathrm{mmol} / \mathrm{l}$ at two hours and $5.0 \mathrm{~mm} / \mathrm{L}$ at $8 \mathrm{~h}$ since last caloric intake in a study of about 28,000 primary care patients [11] and that higher mean random glucose levels are mainly seen in the first $3 \mathrm{~h}$ after a caloric intake which is comparable to our findings. A study of non-glucoseintolerant adults (i.e. also without diabetes) with existing CVD drawn from the Framingham Heart Study (FHS) showed only little differences in age- and sex-adjusted percentiles between random glucose and fasting glucose [25].

In our study, additional adjustment for fasting time did not alter substantially the random glucose-mortality relation. Regarding other endpoints, a study from Asia and the Pacific region had data on both random and fasting glucose and revealed weaker estimates regarding stroke but comparable estimates regarding ischemic heart disease (IHD) for random compared to fasting glucose [26].

\section{Limitations and strengths}

Our study has some limitations which are described in the following. First, although the study population and the overall number of fatal cases were large, the number of cases was rather low for very low and high glucose levels leading to wide confidence intervals; thus, findings should be interpreted with caution for random glucose values at the tails of the distribution. Second, random glucose was measured in serum in contrast to several other studies where blood plasma was used. However, glucose values measured in serum or plasma seem to differ only slightly with lower values in serum which may be considered as not physiologically relevant [27]. Third, random glucose was measured only at the baseline examination; thus potential changes in random glucose levels at the individual level per participant during the follow-up time could not be assessed. Fourth, though non-response in our study was addressed by using survey weights which may compensate under-represented groups (e.g. older aged or low educated), a possible selection bias due to non-response cannot be excluded. Fifth, about $12 \%$ of participant had to be excluded due to missing information in at least one of used variables which may lead also to selection bias. However, a sensitivity analysis applying multiple imputation revealed comparable findings as in the main analyses indicating a rather low selection bias due to missing information. Sixth, residual confounding cannot be excluded, especially regarding further mortality-related risk factors and subclinical diseases which could not be taken into account in the present analyses.

A major strength of the present study is that it was based on a large population-based sample representative of the adult population in Germany with standardized and quality controlled data ascertainment. The mortality follow-up was almost complete with only $2 \%$ of participants lost to follow-up; furthermore, the mean follow-up time of almost 12 years was rather long.

\section{Conclusions}

The present study conducted in a nationally representative study population indicated a significant association 
of random glucose levels with all-cause mortality in adults without known diabetes, even after controlling for mortality-related risk factors, $\mathrm{HbA}_{1 \mathrm{c}}$ and fasting time. Surprisingly, the correlation between random glucose level and fasting time was non-monotonic and weak.

Undoubtedly, measuring fasting glucose or $\mathrm{HbA}_{1 \mathrm{c}}$ have the advantage of being more precise in assessing diabetes risk and diagnosis. However, regarding the value for the assessment of mortality risks in epidemiological studies with large sample size, fasting glucose, $\mathrm{HbA}_{1 \mathrm{c}}$ and random glucose seem to be comparable. The advantage of random glucose consists in its easy assessment independent of the fasting state in contrast to fasting glucose and is connected with lower costs compared to $\mathrm{HbA}_{1 \mathrm{c}}$ which makes it a suitable marker in specific situations. This is particularly true for large population-based health studies, since fasting blood sampling after an overnight fasting period may jeopardize participation rates.

In conclusion, our findings add to existing evidence that random glucose is a useful tool for assessing health risks among people without diagnosed diabetes, especially when fasting glucose or $\mathrm{HbA}_{1 \mathrm{c}}$ is difficult to obtain.

\section{Additional file}

Additional file 1: Figure S1. The box plot gives information about distribution measures of random glucose levels stratified by fasting time categories: the full range from minimum to maximum (at bottom and top of the whiskers), the interquartile range from lower to upper quartile (at bottom and top of the boxes), the median (straight line inside the boxes) and the mean (point inside the boxes); the median values in the fasting time categories are connected by a solid line. (DOCX $30 \mathrm{~kb}$ )

\section{Abbreviations}

BMI: Body mass index; CAPI: Computer-assisted personal interview; CASMIN: Comparative Analysis of Social Mobility in Industrial Nations; Cl: Confidence interval; CVD: Cardiovascular disease; DEGS1: German Health Interview and Examination Survey for Adults; FHS: Framingham Heart Study GNHIES98: German National Health Interview and Examination Survey 1998; $\mathrm{HbA}_{1 \mathrm{c}}$ : Glycated hemoglobin $\mathrm{A}_{1 \mathrm{c}}$;HR: Hazard ratio; RKI: Robert Koch Institute

\section{Acknowledgements}

The authors would like to thank Ingrid-Katharina Wolf and Michael Lange from the Robert Koch Institute for conducting the mortality follow-up and Wulf Thierfelder and Thea Riedel for overseeing the laboratory analyses and quality assurance of random glucose measurements in the Robert Koch Institute Central Epidemiological Laboratory.

\section{Funding}

This work was supported by a research grant from the Federal Ministry of Health, Germany, to develop a diabetes surveillance system in Germany (Grant Number: GE 201503 23). Christa Scheidt-Nave reports grants from the Federal Ministry of Health, Germany (Grant Number: ZMVI1-2515-FSB-782), during the conduct of the study. The conduction of national health surveys in Germany is funded by the Federal Ministry of Health as part of the continuous national health monitoring.

The funding bodies stated above had no role in either the design of the study, the collection, analysis, and interpretation of data or in writing the manuscript.

\section{Availability of data and materials}

The authors confirm that some access restrictions apply to the data underlying the findings. The data set cannot be made publicly available because informed consent from study participants did not cover public deposition of data. However, the minimal data set underlying the findings is archived in the 'Health Monitoring' Research Data Centre at the Robert Koch Institute (RKI) and can be accessed by all interested researchers. On-site access to the data set is possible at the Secure Data Center of the RKI's 'Health Monitoring' Research Data Centre. Requests should be submitted to the 'Health Monitoring' Research Data Centre, Robert Koch Institute, Berlin, Germany (e-mail: fdz@rki.de)

\section{Authors' contributions}

JB performed the statistical analyses and drafted the manuscript. $\mathrm{CH}, \mathrm{RP}$ and YD made substantial contributions to the interpretation of data. CSN had the idea for conducting the study and made substantial contributions to the interpretation of data. All authors critically revised the manuscript for important intellectual content and read and approved the final version of the manuscript.

\section{Ethics approval and consent to participate}

The GNHIES98 and its mortality follow-up were approved by the Federal Commissioner for the Protection of Data and Freedom of Information (Germany). All participants provided written informed consent before enrolment.

\section{Consent for publication}

Not applicable.

\section{Competing interests}

The authors declare that they have no competing interests.

\section{Publisher's Note}

Springer Nature remains neutral with regard to jurisdictional claims in published maps and institutional affiliations.

Received: 17 May 2018 Accepted: 26 November 2018

Published online: 13 December 2018

References

1. van Dieren S, Beulens JW, van der Schouw YT, Grobbee DE, Neal B. The global burden of diabetes and its complications: an emerging pandemic. Eur J Cardiovasc Prev Rehabil. 2010;17(1):S3-8.

2. Rao Kondapally Seshasai S, Kaptoge S, Thompson A, Di Angelantonio E, Gao $P$, Sarwar N, et al. Diabetes mellitus, fasting glucose, and risk of causespecific death. N Engl J Med. 2011;364(9):829-41.

3. Tancredi M, Rosengren A, Svensson AM, Kosiborod M, Pivodic A, Gudbjornsdottir S, et al. Excess mortality among persons with type 2 diabetes. N Engl J Med. 2015;373(18):1720-32.

4. World Health Organization. Gobal report on diabetes. Geneva: World Health Organization; 2016.

5. Huang Y, Cai X, Mai W, Li M, Hu Y. Association between prediabetes and risk of cardiovascular disease and all cause mortality: systematic review and meta-analysis. BMJ. 2016;355:15953.

6. Sarwar N, Gao P, Seshasai SR, Gobin R, Kaptoge S, Emerging Risk Factors Collaboration, et al. Diabetes mellitus, fasting blood glucose concentration, and risk of vascular disease: a collaborative meta-analysis of 102 prospective studies. Lancet. 2010;375(9733):2215-22.

7. Park C, Guallar E, Linton JA, Lee DC, Jang Y, Son DK, et al. Fasting glucose level and the risk of incident atherosclerotic cardiovascular diseases. Diabetes Care. 2013;36(7):1988-93.

8. Paprott R, Schaffrath Rosario A, Busch MA, Du Y, Thiele S, Scheidt-Nave C, et al. Association between hemoglobin A1C and all-cause mortality: results of the mortality follow-up of the German National Health Interview and examination survey 1998. Diabetes Care. 2015;38(2):249-56.

9. Cavero-Redondo I, Peleteiro B, Alvarez-Bueno C, Rodriguez-Artalejo F, Martinez-Vizcaino V. Glycated haemoglobin A1c as a risk factor of cardiovascular outcomes and all-cause mortality in diabetic and nondiabetic populations: a systematic review and meta-analysis. BMJ Open. 2017;7(7):e015949

10. NCDRF collaboration. Effects of diabetes definition on global surveillance of diabetes prevalence and diagnosis: a pooled analysis of 96 populationbased studies with 331,288 participants. Lancet Diabetes Endocrinol. 2015; 3(8):624-37. 
11. Moebus S, Gores L, Losch C, Jockel KH. Impact of time since last caloric intake on blood glucose levels. Eur J Epidemiol. 2011;26(9):719-28.

12. Kowall B, Rathmann W, Giani G, Schipf S, Baumeister S, Wallaschofski H, et al. Random glucose is useful for individual prediction of type 2 diabetes: results of the study of health in Pomerania (SHIP). Prim Care Diabetes. 2013; 7(1):25-31.

13. Bowen ME, Xuan L, Lingvay I, Halm EA. Random blood glucose: a robust risk factor for type 2 diabetes. J Clin Endocrinol Metab. 2015;100(4):1503-10.

14. Bragg F, Li L, Bennett D, Guo Y, Lewington S, Bian Z, et al. Association of Random Plasma Glucose Levels with the risk for cardiovascular disease among Chinese adults without known diabetes. JAMA Cardiol. 2016;1(7):813-23.

15. Rosness TA, Engedal K, Bjertness E, Strand BH. Association between random measured glucose levels in middle and old age and risk of dementiarelated death. J Am Geriatr Soc. 2016;64(1):156-61.

16. Thefeld W, Stolzenberg H, Bellach BM. The Federal Health Survey: response, composition of participants and non-responder analysis]. Gesundheitswesen 1999;61 Spec No:S57-S61.

17. Wolf IK, Busch M, Lange M, Kamtsiuris P, Doelle R, Richter A, et al. Mortality follow-up of the German health interview and examination survey for adults (DEGS) : methods and first results. Bundesgesundheitsblatt Gesundheitsforschung Gesundheitsschutz. 2014;57(11):1331-7.

18. Brauns HSS, Steinmann S. The CASMIN educational classification in international comparative research. In: Hoffmeyer-Zlotnik JHWC, editor. Advances in cross-National Comparison. New York: Kluwer; 2003. p. 221-44.

19. Burger M, Mensink G, Bronstrup A, Thierfelder W, Pietrzik K. Alcohol consumption and its relation to cardiovascular risk factors in Germany. Eur J Clin Nutr. 2004;58(4):605-14.

20. Heidemann C, Scheidt-Nave C, Richter A, Mensink GB. Dietary patterns are associated with cardiometabolic risk factors in a representative study population of German adults. Br J Nutr. 2011;106(8):1253-62.

21. Aggarwal V, Schneider AL, Selvin E. Low hemoglobin a(1c) in nondiabetic adults: an elevated risk state? Diabetes Care. 2012;35(10):2055-60.

22. Berglund P. Multiple imputation using the fully conditional specification method: a comparison of SAS Stata, IVEware, and R; 2015.

23. von Elm E, Altman DG, Egger M, Pocock SJ, Gotzsche PC, Vandenbroucke $J P$, et al. The strengthening the reporting of observational studies in epidemiology (STROBE) statement: guidelines for reporting observational studies. J Clin Epidemiol. 2008;61(4):344-9.

24. Staiano AE, Reeder BA, Elliott S, Joffres MR, Pahwa P, Kirkland SA, et al. Body mass index versus waist circumference as predictors of mortality in Canadian adults. Int J Obes. 2012;36(11):1450-4.

25. Port SC, Goodarzi MO, Boyle NG, Jennrich Rl. Blood glucose: a strong risk factor for mortality in nondiabetic patients with cardiovascular disease. Am Heart J. 2005;150(2):209-14

26. Lawes CM, Parag V, Bennett DA, Suh I, Lam TH, Whitlock G, et al. Blood glucose and risk of cardiovascular disease in the Asia Pacific region. Diabetes Care. 2004;27(12):2836-42.

27. Frank EA, Shubha MC, D'Souza CJ. Blood glucose determination: plasma or serum? J Clin Lab Anal. 2012;26(5):317-20.

Ready to submit your research? Choose BMC and benefit from:

- fast, convenient online submission

- thorough peer review by experienced researchers in your field

- rapid publication on acceptance

- support for research data, including large and complex data types

- gold Open Access which fosters wider collaboration and increased citations

- maximum visibility for your research: over $100 \mathrm{M}$ website views per year

At BMC, research is always in progress.

Learn more biomedcentral.com/submissions 